\title{
Reviewer Acknowledgements for Global Journal of Health Science, Vol. 14, No. 2
}

Global Journal of Health Science wishes to acknowledge the following individuals for their assistance with peer review of manuscripts for this issue. Their help and contributions in maintaining the quality of the journal are greatly appreciated.

Global Journal of Health Science is recruiting reviewers for the journal. If you are interested in becoming a reviewer, we welcome you to join us. Please contact us for the application form at: gjhs@ccsenet.org.

\section{Reviewers for Volume 14, Number 2}

Farahnaz Amini, UCSI University, Malaysia

Fathi Shamma, The Arab Academic College Israel Haifa, Israel

France Ncube, Bindura University of Science Education, Zimbabwe

Francisco Rodenas Rigla, University of Valencia, Spain

Gabriel Gulis, University of Southern Denmark, Denmark

Gavric Zivana, University Banja Luka, Bosnia and Herzegovina

Georgann Valerie Weissman, Capella University, United States of America

Gunta Beta, Riga Stradins University, Latvia

Loray Daws, British Columbia Masterson Institute, Canada

Marcel Wullschleger, University of Bern, Switzerland

Maria Malliarou, University of Thessaly, Greece

Meng Zhao, Texas A\&M University at Corpus Christi, United States of America

Misheck Dube, North West University, South Africa

Pedram Iranmanesh, Dentist, Independent Researcher, Iran

Pi-Ming Yeh, Missouri Western State University, United States of America

Piotr Raźniak, Pedagogical University, Poland

Raúl Quevedo-Blasco, Universidad de Granada, Spain

Thammanard Charernboon, Thammasat University, Thailand

Thanusin Saleeon, Mae Fah Lung University, Thailand

Tulyakul Phatcharapon, Boromarajonani College of Nursing, Thailand

Zohreh Vanaki, Tarbiat Modares University, Iran 